\title{
Explicit Stochastic Nonlinear Predictive Control Based on Gaussian Process Models
}

\author{
Alexandra Grancharova, Juš Kocijan, and Tor A. Johansen
}

\begin{abstract}
Nonlinear Model Predictive Control (NMPC) algorithms are based on various nonlinear models. Recently, an on-line optimization approach for stochastic NMPC based on a Gaussian process model was proposed. A significant advantage of the Gaussian process models is that they provide information about prediction uncertainties, which would be of help in NMPC design. On the other hand, an explicit solution to the stochastic NMPC problem based on Gaussian process model would allow efficient on-line computations as well as verifiability of the implementation. This paper suggests an approximate multi-parametric Nonlinear Programming approach to explicit solution of stochastic NMPC problems for constrained nonlinear systems based on Gaussian process model. In particular, the reference tracking problem is considered. The approach builds an orthogonal search tree structure of the state space partition and consists in constructing a feasible PWL approximation to the optimal control sequence.
\end{abstract}

\section{INTRODUCTION}

$\mathrm{N}$ ONLINEAR Model Predictive Control (NMPC) involves the solution at each sampling instant of a finite horizon optimal control problem subject to nonlinear system dynamics and state and input constraints [1], [2], [3], [4]. A recent survey of the main on-line optimization strategies of NMPC is given in [5]. Mathematical models of engineering systems usually contain some amount of uncertainty (unknown additive disturbances and/or uncertain model parameters). Therefore, the robust MPC problem formulation requires the model uncertainty to be taken into account. In many applications, the system to be controlled is described by a stochastic model where the probabilistic distribution of the uncertainty is assumed to be known. Several approaches for constrained MPC based on stochastic models (stochastic MPC) are proposed in [6]-[12]. The approaches [6], [7], [8] are based on linear state space

This work was sponsored by the Ad-Futura science and education foundation of Republic of Slovenia and by the Research Council of Norway through the Strategic University Programme on Computational Methods in Nonlinear Motion Control

A. Grancharova is with the Institute of Control and System Research, Bulgarian Academy of Sciences, Acad. G. Bonchev str., B1.2, P.O.Box 79, Sofia 1113, Bulgaria (phone: 3592-870-03-37; fax: 3592-870-33-61; e-mail: alexandra.grancharova@abv.bg).

J. Kocijan is with the Department of Systems and Control, Jozef Stefan Institute, Jamova 39, 1000 Ljubljana, Slovenia and with University of Nova Gorica, School of Engineering and Management, Vipavska 13, 5000 Nova Gorica, Slovenia (e-mail: jus.kocijan@ijs.si).

T. A. Johansen is with the Department of Engineering Cybernetics, Norwegian University of Science and Technology, 7491 Trondheim, Norway (e-mail: Tor.Arne.Johansen@itk.ntnu.no). models with stochastic parameters and/or additive noise and they optimize the expected value of the cost function subject to hard input constraints [6] or probabilistic constraints [7], [8]. In [9], [10], [11], [12], stochastic MPC approaches incorporating a probabilistic cost and probabilistic constraints are developed. The method suggested in [9] is based on a moving average (MA) model with random coefficients. It was further extended to linear time-varying MA models [10] and to state space models with stochastic uncertainty in the output or the input map [11], [12].

It should be noted that the stochastic MPC approaches [6]-[12] are based on parametric probabilistic models. Alternatively, the stochastic systems can be modeled with non-parametric models which can offer a significant advantage compared to the parametric models. This is related to the fact that the non-parametric probabilistic models provide information about prediction uncertainties which are difficult to evaluate appropriately with the parametric models. The Gaussian process model is an example of a non-parametric probabilistic black-box model and up to now it has been applied to model mainly static nonlinearities. The use of Gaussian processes in the modelling of dynamic systems is a recent development e.g. [13], [14], [15]. In [16], [17], [18], an on-line optimization approach for stochastic NMPC based on Gaussian process model is proposed.

It has recently been shown that the feedback solution to linear and quadratic constrained MPC problems has an explicit representation as a piece-wise linear (PWL) state feedback defined on a polyhedral partition of the state space [19]. The benefits of an explicit solution, in addition to the efficient on-line computations, include also verifiability of the implementation, which is an essential issue in safetycritical applications. For nonlinear and stochastic MPC the prospects of explicit solutions are even higher than for linear MPC, since the benefits of computational efficiency and verifiability are even more important. An approach for efficient on-line computation of NMPC for constrained input-affine nonlinear systems has been suggested in [20]. In [21], [22], [23], approaches for off-line computation of explicit sub-optimal PWL predictive controllers for general nonlinear systems with state and input constraints have been developed, based on the multi-parametric Nonlinear Programming (mp-NLP) ideas [24]. The mentioned methods for explicit NMPC are based on deterministic first principle models of the systems. 
This paper suggests an approximate mp-NLP approach to explicit solution of stochastic NMPC problems for constrained nonlinear systems based on a Gaussian process model (referred to as GP-NMPC problems). In particular, the reference tracking problem is considered. The contribution of the present work is the formulation of a more general GP-NMPC problem compared to [16], [17], [18], and representing it as an mp-NLP problem. Further, the approximate mp-NLP approach [23] is applied to build an orthogonal search tree structure of the state space partition and construct a feasible PWL approximation to the optimal control sequence. Thus, the approach proposed in this paper can be considered as an application of the approximate methods [22], [23], [25] for explicit solution of MPC problems to the cases where the system dynamics is described by a probabilistic (Gaussian process) model.

The following notation will be used in the paper. $A \succ 0$ means that the square matrix $A$ is positive definite. For $x \in \mathbb{R}^{n}$, the Euclidean norm is $\|x\|=\sqrt{x^{T} x}$ and the weighted norm is defined for some symmetric matrix $A \succ 0$ as $\|x\|_{A}=\sqrt{x^{T} A x}$. For a random variable $y$ with Gaussian distribution, $\mathcal{N}\left(\mu(y), \sigma^{2}(y)\right)$ denotes its probability distribution, and $\mu(y)$ and $\sigma^{2}(y)$ are respectively its mean and variance.

\section{MODELLING OF DYNAMIC SYSTEMS WITH GAUSSIAN PROCESSES}

A Gaussian process is an example of the use of a flexible, probabilistic, nonparametric model which directly provides us with uncertainty predictions. Its use and properties for modelling are reviewed in [26].

A Gaussian process is a collection of random variables which have a joint multivariate Gaussian distribution. Assuming a relationship of the form $y=f(z)$ between an input $z \in \mathbb{R}^{D}$ and output $y \in \mathbb{R}$, we have $y(1), y(2), \ldots, y(M) \sim \mathcal{N}(0, \mathbf{\Sigma})$, where $\Sigma_{p q}=\operatorname{Cov}(y(p), y(q))=C(z(p), z(q))$ gives the covariance between the output points $y(p)$ and $y(q)$ corresponding to the input points $z(p)$ and $z(q)$. Thus, the mean $\mu(z)$ (usually assumed to be zero) and the covariance function $C(z(p), z(q))$ fully specify the Gaussian process. Note that the covariance function $C(z(p), z(q))$ can be any function with the property that it generates a positive definite covariance matrix. A common choice is:

$$
C(z(p), z(q))=v_{1} \exp \left[-\frac{1}{2} \sum_{i=1}^{D} w_{i}\left(z_{i}(p)-z_{i}(q)\right)^{2}\right]+v_{0} \alpha_{p q}(1)
$$

where $\Theta=\left[w_{1}, \ldots, w_{D}, v_{0}, v_{1}\right]$ are the 'hyperparameters' of the covariance function, $z_{i}$ denotes the $i$-th component of the $D$-dimensional input vector $z$, and $\alpha_{p q}$ is the Kronecker operator. Other forms of covariance functions suitable for different applications can be found in [27]. For a given problem, the hyperparameters are learned (identified) using the data at hand. After the learning, one can use the $w$ parameters as indicators of 'how important' the corresponding input components (dimensions) are: if $w_{i}$ is zero or near zero it means that the inputs in dimension $i$ contain little information and could possibly be removed.

Consider a set of $M D$-dimensional input vectors $\mathbf{Z}=[z(1), z(2), \ldots, z(M)]^{T}$ and a vector of output data $Y=[y(1), y(2), \ldots, y(M)]^{T}$. Based on the data $(\mathbf{Z}, Y)$, and given a new input vector $z^{*}$, we wish to estimate the probability distribution of the corresponding output $y^{*}$. Unlike other models, there is no model parameter determination as such, within a fixed model structure. With this model, most of the effort consists in tuning the parameters of the covariance function. This is done by maximizing the log-likelihood of the parameters, which is computationally relatively demanding since the inverse of the data covariance matrix $(M \times M)$ has to be calculated at every iteration.

The described approach can be easily utilized for regression calculation. Based on a training set $\mathbf{Z}$, a covariance matrix $\mathbf{K}$ of size $M \times M$ is determined. As already mentioned before, the aim is to estimate the probability distribution of the corresponding output $y^{*}$ at some new input vector $z^{*}$. For a new test input $z^{*}$, the predictive distribution of the corresponding output is $y^{*} \mid z^{*},(\mathbf{Z}, Y)$ and is Gaussian, with mean and variance:

$$
\begin{aligned}
& \mu\left(z^{*}\right)=k\left(z^{*}\right)^{T} \mathbf{K}^{-1} Y \\
& \sigma^{2}\left(z^{*}\right)=k_{0}\left(z^{*}\right)-k\left(z^{*}\right)^{T} \mathbf{K}^{-1} k\left(z^{*}\right)+v_{0}
\end{aligned}
$$

where $k\left(z^{*}\right)=\left[C\left(z(1), z^{*}\right), \ldots, C\left(z(M), z^{*}\right)\right]^{T}$ is the $M \times 1$ vector of covariances between the test and training cases and $k_{0}\left(z^{*}\right)=C\left(z^{*}, z^{*}\right)$ is the covariance between the test input and itself.

Gaussian processes can be used to model static nonlinearities and can therefore be used for modelling of dynamic systems if delayed input and output signals are used as regressors [13]. In such cases an autoregressive model is considered, such that the current predicted output depends on previous estimated outputs, as well as on previous control inputs:

$$
\begin{gathered}
z(t)=[\hat{y}(t-1), \hat{y}(t-2), \ldots, \hat{y}(t-L), u(t-1), \\
u(t-2), \ldots, u(t-L)]^{T} \\
\hat{y}(t)=f(z(t))+\eta(t)
\end{gathered}
$$

where $t$ denotes consecutive number of data sample, $L$ is a given lag, and $\eta(t)$ is the prediction error. The quality of the predictions with a Gaussian process model is assessed by 
computing the average squared error (ASE):

$$
A S E=\frac{1}{M} \sum_{i=1}^{M}[\mu(\hat{y}(i))-y(i)]^{2}
$$

and by the log density error (LD) [13]:

$$
L D=\frac{1}{2 M} \sum_{i=1}^{M} \log (2 \pi)+\log \left[\sigma^{2}(\hat{y}(i))\right]+\frac{[\mu(\hat{y}(i))-y(i)]^{2}}{\sigma^{2}(\hat{y}(i))}
$$

In (4), (5), $\mu(\hat{y}(i))$ and $\sigma^{2}(\hat{y}(i))$ are the prediction mean and variance, $y(i)$ is the system's output and $M$ is the number of the training points.

The iterative multi-step ahead prediction can be done in the following ways, as described in [28]:

1) by feeding back at each time step the predictive mean only;

2) by feeding back at each time step both the predictive mean and the predictive variance;

3) by Monte Carlo simulations.

Thus, the uncertainty attached to each intermediate prediction is taken into account. The Gaussian process model now not only describes the dynamic characteristics of the non-linear system, but at the same time provides information about the confidence in the predictions. The Gaussian process can highlight areas of the input space where prediction quality is poor, due to the lack of data, by indicating the higher variance around the predicted mean. It is worthwhile noting that the derivatives of means and variances with respect to input data can be calculated in straightforward manner. For more details see [28].

\section{FORMULATION OF THE GP-NMPC PROBLEM AS AN MP- NLP PROBLEM}

Consider a stochastic system described by an uncertain nonlinear discrete-time model:

$$
x(t+1)=f(x(t), u(t))+\xi(t)
$$

where $x(t) \in \mathbb{R}^{n}$ and $u(t) \in \mathbb{R}^{m}$ are the state and input variables, $\xi(t) \in \mathbb{R}^{n}$ are Gaussian disturbances, and $f: \mathbb{R}^{n} \times \mathbb{R}^{m} \rightarrow \mathbb{R}^{n}$ is a nonlinear continuous function. The uncertainty consists in that the analytical expression of $f(x, u)$ is not known and neither are the mean values and the covariances of the disturbances $\xi(t)$. The relationship (6) is represented in the form:

$$
y(t)=f_{G}(z(t))+\xi(t)
$$

where $y(t)=x(t+1) \in \mathbb{R}^{n}$ and $z(t)=[x(t), u(t)] \in \mathbb{R}^{n+m}$. Suppose that we have an output data set $Y_{i}=\left[y_{i}(0), y_{i}(1), \ldots, y_{i}(M-1)\right], \quad i=1,2, \ldots, n$ corresponding to an input data set $\mathbf{Z}=[z(0), z(1), \ldots, z(M-1)]$. Assume that the relationship (7) is approximated with Gaussian processes with distributions:

$$
Y_{1} \sim \mathcal{N}\left(0, \Sigma_{1}\right), Y_{2} \sim \mathcal{N}\left(0, \Sigma_{2}\right), \ldots, Y_{n} \sim \mathcal{N}\left(0, \Sigma_{n}\right)
$$

where the covariance functions
$\Sigma_{1, p q}=\operatorname{Cov}_{1}\left(y_{1}(p), y_{1}(q)\right)=C_{1}(z(p), z(q))$,

$\Sigma_{n, p q}=\operatorname{Cov}_{n}\left(y_{n}(p), y_{n}(q)\right)=C_{n}(z(p), z(q))$

with

$p=0,1, \ldots, M-1, q=0,1, \ldots, M-1$, depend on the given input and output data sets. Having obtained the Gaussian process model (8), the probability distribution of the output $y(M)$ corresponding to a new input $z(M)$ can be determined as described in the previous section:

$$
\begin{aligned}
& y_{1}(M) \mid z(M),\left(\mathbf{Z}, Y_{1}\right) \sim \mathcal{N}\left(\mu\left(y_{1}(M)\right), \sigma^{2}\left(y_{1}(M)\right)\right) \\
& \vdots \\
& y_{n}(M) \mid z(M),\left(\mathbf{Z}, Y_{n}\right) \sim \mathcal{N}\left(\mu\left(y_{n}(M)\right), \sigma^{2}\left(y_{n}(M)\right)\right)
\end{aligned}
$$

In (9), $\mu\left(y_{i}(M)\right)$ and $\sigma^{2}\left(y_{i}(M)\right)$ denote respectively the mean and the variance of the output variable $y_{i}(M)$, $i=1,2, \ldots, n$. We introduce the vectors

$\mu_{y}(M)=\left[\mu\left(y_{1}(M)\right), \ldots, \mu\left(y_{n}(M)\right)\right]$

and

$\sigma_{y}^{2}(M)=\left[\sigma^{2}\left(y_{1}(M)\right), \ldots, \sigma^{2}\left(y_{n}(M)\right)\right]$ and the matrix $\mathbf{Y}=\left[Y_{1}, Y_{2}, \ldots, Y_{n}\right]$. Then, the relation (9) is represented:

$$
y(M) \mid z(M),(\mathbf{Z}, \mathbf{Y}) \sim \mathcal{N}\left(\mu_{y}(M), \sigma_{y}^{2}(M)\right)
$$

As shown in [28], it is possible to obtain a multi-step ahead prediction:

$$
\begin{aligned}
& y(M+k) \mid z(M+k),(\mathbf{Z}, \mathbf{Y}) \sim \mathcal{N}\left(\mu_{y}(M+k), \sigma_{y}^{2}(M+k)\right) \\
& k=0,1, \ldots, N-1
\end{aligned}
$$

Suppose the initial state $x(t)=x_{t \mid t}$ and the control inputs $u(t+k)=u_{t+k}, k=0,1, \ldots, N-1$ are given. Then, by taking into account that $y(t)=x(t+1)$ and $z(t)=[x(t), u(t)]$, from (11) we obtain the probability distribution of the predicted states $x_{t+k+1 \mid t}, k=0,1, \ldots, N-1$ which correspond to the given initial state $x_{t \mid t}$ and control inputs $u_{t+k}, k=0,1, \ldots, N-1$ :

$$
\begin{aligned}
& x_{t+k+1 \mid t} \mid x_{t+k \mid t}, u_{t+k} \sim \mathcal{N}\left(\mu\left(x_{t+k+1 \mid t}\right), \sigma^{2}\left(x_{t+k+1 \mid t}\right)\right) \\
& k=0,1, \ldots, N-1
\end{aligned}
$$

The $95 \%$ confidence interval of the random variable $x_{t+k+1 \mid t}$ is $\left[\mu\left(x_{t+k+1 \mid t}\right)-2 \sigma\left(x_{t+k+1 \mid t}\right) ; \mu\left(x_{t+k+1 \mid t}\right)+2 \sigma\left(x_{t+k+1 \mid t}\right)\right]$, where $\sigma\left(x_{t+k+1 \mid t}\right)$ is the standard deviation.

Here, we consider a reference tracking problem where the goal is to have the state vector $x(t)$ track the reference signal $r(t) \in \mathbb{R}^{n}$. In the problem formulation, the type of the cost function is like the one used in [19]. Suppose that a full measurement of the state $x(t)$ is available at the current time $t$. For the current $x(t)$, the reference tracking GP-NMPC solves the following optimization problem:

\section{Problem P1:}

$$
V^{*}(x(t), r(t), u(t-1))=\min _{U} J(U, x(t), r(t), u(t-1))
$$

subject to $x_{t \mid t}=x(t)$ and: 


$$
\begin{aligned}
& \mu\left(x_{t+k \mid t}\right)-2 \sigma\left(x_{t+k \mid t}\right) \geq x_{\min }, k=1, \ldots, N \\
& \mu\left(x_{t+k \mid t}\right)+2 \sigma\left(x_{t+k \mid t}\right) \leq x_{\max }, k=1, \ldots, N \\
& u_{\min } \leq u_{t+k} \leq u_{\max }, k=0,1, \ldots, N-1 \\
& \Delta u_{\min } \leq \Delta u_{t+k} \leq \Delta u_{\max }, k=0,1, \ldots, N-1 \\
& \max \left\{\left\|\mu\left(x_{t+N \mid t}\right)-2 \sigma\left(x_{t+N \mid t}\right)-r(t)\right\|,\right. \\
& \left.\quad\left\|\mu\left(x_{t+N \mid t}\right)+2 \sigma\left(x_{t+N \mid t}\right)-r(t)\right\|\right\} \leq \delta \\
& \Delta u_{t+k}=u_{t+k}-u_{t+k-1}, k=0,1, \ldots, N-1 \\
& x_{t+k+1 \mid t} \mid x_{t+k \mid t}, u_{t+k} \sim \mathcal{N}\left(\mu\left(x_{t+k+1 \mid t}\right), \sigma^{2}\left(x_{t+k+1 \mid t}\right)\right) \\
& k=0,1, \ldots, N-1
\end{aligned}
$$

with $U=\left[u_{t}, u_{t+1}, \ldots, u_{t+N-1}\right]$ and the cost function given by:

$$
\begin{array}{r}
J(U, x(t), r(t), u(t-1))=\left\|\mu\left(x_{t+N \mid t}\right)-r(t)\right\|_{P}^{2}+ \\
\sum_{k=0}^{N-1}\left[\left\|\mu\left(x_{t+k \mid t}\right)-r(t)\right\|_{Q}^{2}+\left\|\Delta u_{t+k}\right\|_{R}^{2}\right]
\end{array}
$$

Here, $N$ is a finite horizon. From a stability point of view it is desirable to choose $\delta$ in (18) as small as possible [2]. However, due to the uncertainty of the $x_{t+N \mid t}$ prediction, characterized by the variance $\sigma^{2}\left(x_{t+N \mid t}\right)$, the feasibility of problem P1 will rely on $\delta$ being sufficiently large. A part of the GP-NMPC design will be to address this tradeoff. If the system is asymptotically stable (or pre-stabilized), $N$ is large, and the Gaussian process model has a small prediction uncertainty, then it is more likely that the choice of a small $\delta$ will be possible.

A more general stochastic MPC problem is formulated in [9], [10], [11], [12], where a probabilistic formulation of the cost is introduced that includes the probabilistic bounds of the predicted variable. Also in these references, a probabilistic formulation of the constraints is used, i.e. the random variable should not exceed a certain bound with a given probability. The stochastic MPC problem considered in this paper (problem P1) is of a more special form compared to the general problem formulated in [9]-[12]. Here, the cost function (21) includes the mean value of the random variable and the constraints (14), (15) and (18) are equivalent to the following probabilistic constraints:

$$
\begin{aligned}
& \operatorname{Pr}\left(x_{t+k \mid t} \geq x_{\min }\right)=p, k=1, \ldots, N \\
& \operatorname{Pr}\left(x_{t+k \mid t} \leq x_{\max }\right)=p, k=1, \ldots, N \\
& \operatorname{Pr}\left(\left\|x_{t+N \mid t}-r(t)\right\| \leq \delta\right)=p
\end{aligned}
$$

where the probability $p$ is 0.95 (the confidence interval used in (14), (15) and (18) is associated with this level of probability).

The following assumptions are made:

A1. $P, Q, R \succ 0$.

A2. $x_{\text {min }}<0<x_{\text {max }}, u_{\text {min }}<0<u_{\text {max }}$ and $\Delta u_{\text {min }}<0<\Delta u_{\text {max }}$.

We introduce an extended state vector:

$$
\tilde{x}(t)=[x(t), r(t), u(t-1)] \in \mathbb{R}^{\tilde{n}}, \tilde{n}=2 n+m
$$

Let $\tilde{x}$ be the value of the extended state at the current time $t$. Then, the optimization problem P1 can be formulated in a compact form as follows:

Problem P2:

$$
V^{*}(\tilde{x})=\min _{U} J(U, \tilde{x})
$$

subject to:

$$
G(U, \tilde{x}) \leq 0
$$

The GP-NMPC problem defines an mp-NLP, since it is NLP in $U$ parameterized by $\tilde{x}$. An optimal solution to this problem is denoted $U^{*}=\left[u_{t}^{*}, u_{t+1}^{*}, \ldots, u_{t+N-1}^{*}\right]$ and the control input is chosen according to the receding horizon policy $u(t)=u_{t}^{*}$. Define the set of $N$-step feasible initial states as follows:

$$
X_{f}=\left\{\tilde{x} \in \mathbb{R}^{\tilde{n}} \mid G(U, \tilde{x}) \leq 0 \text { for some } U \in \mathbb{R}^{N m}\right\}
$$

If $\delta$ in (18) is chosen such that the problem P1 is feasible, then $X_{f}$ is a non-empty set and due to assumption A2, the origin is an interior point in $X_{f}$.

In parametric programming problems one seeks the solution $U^{*}(\tilde{x})$ as an explicit function of the parameters $\tilde{x}$ in some set $X \subseteq X_{f} \subseteq \mathbb{R}^{\tilde{n}}$ [24]. The explicit solution allows us to replace the computationally expensive real-time optimization with a simple function evaluation. In this paper we suggest a computational method for constructing an explicit PWL approximate solution of the reference tracking GP-NMPC problem.

\section{APPROXIMATE MP-NLP APPROACH TO EXPLICIT GP- NMPC}

Here, the computational issues related to the nonconvexity of the optimization problem are treated in a way similar to that in [23].

\section{A. Close-to-global solution of mp-NLP}

In general, problem P2 can be non-convex with multiple local minima. Therefore, it would be necessary to apply an efficient initialization of problem P2 so to find a close-toglobal solution. One possible way to obtain this is to find a close-to-global solution at a point $v_{0} \in X_{0}$ by comparing the local minima corresponding to several initial guesses and then to use this solution as an initial guess at the neighbouring points $v_{i} \in X_{0}, i=1,2, \ldots, N_{1}$, i.e. to propagate the solution. The following procedure is used to generate a set of points $V_{0}=\left\{v_{0}, v_{1}, v_{2}, \ldots, v_{N_{1}}\right\}$, where $v_{i} \in X_{0}, i=0,1,2, \ldots, N_{1}$.

\section{Procedure 1 (generation of set of points):}

Consider any hyper-rectangle $X_{0} \subseteq X_{f}$ with vertices $\Lambda^{0}=\left\{\lambda_{1}^{0}, \lambda_{2}^{0}, \ldots, \lambda_{N_{\lambda}}^{0}\right\}$ and center point $v_{0}$. Consider also 
the hyper-rectangles $X_{0}^{j} \subset X_{0}, j=1,2, \ldots, N_{j}$ with vertices respectively

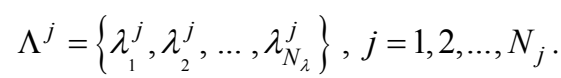

Suppose $X_{0}^{1} \subset X_{0}^{2} \subset \ldots \subset X_{0}^{N_{j}}$. For each of the hyperrectangles $X_{0}$ and $X_{0}^{j} \subset X_{0}, j=1,2, \ldots, N_{j}$, determine a set of points that belongs to its facets and denote this set $\Phi^{j}=\left\{\phi_{1}^{j}, \phi_{2}^{j}, \ldots, \phi_{N_{\phi}}^{j}\right\}, j=0,1,2, \ldots, N_{j}$. Define the set of all points $V_{0}=\left\{v_{0}, v_{1}, v_{2}, \ldots, v_{N_{1}}\right\}$ where $v_{i} \in\left\{\bigcup_{j=0}^{N_{j}} \Lambda^{j}\right\} \bigcup\left\{\bigcup_{j=0}^{N_{j}} \Phi^{j}\right\}, i=1,2, \ldots, N_{1}$.

The following procedure is applied to find a close-toglobal solution at the points $v_{i} \in V_{0}, i=0,1,2, \ldots, N_{1}$ :

Procedure 2 (close-to-global solution of problem P2):

Consider any hyper-rectangle $X_{0} \subseteq X_{f}$ with a set of points $V_{0}=\left\{v_{0}, v_{1}, v_{2}, \ldots, v_{N_{1}}\right\}$ determined by applying Procedure 1. Then:

a). Determine a close-to-global solution of problem $\mathrm{P} 2$ at the center point $v_{0}$ through the following minimization:

$$
U^{*}\left(v_{0}\right)=\arg \min _{U_{i}^{\text {local }} \in\left\{U_{1}^{\text {local }}, \ldots, U_{N_{U}}^{\text {local }}\right\}} J\left(U_{i}^{\text {local }}, v_{0}\right),
$$

where $U_{i}^{\text {local }}, i=1,2, \ldots, N_{U}$ correspond to local minima of the cost function $J\left(U, v_{0}\right)$ obtained for a number of initial guesses $U_{i}^{0}, i=1,2, \ldots, N_{U}$.

b). Determine a close-to-global solution of problem $\mathrm{P} 2$ at the points $v_{i} \in V_{0}, i=1,2, \ldots, N_{1}$ in the following way:

1. Determine a close-to-global solution of $\mathrm{P} 2$ at the center point $v_{0}$ by solving problem (29). Let $i=1$.

2. Let $V^{s}=\left\{v_{0}, v_{1}, v_{2}, \ldots, v_{N_{2}}\right\} \subset V_{0}$ be the subset of points at which a feasible solution of $\mathrm{P} 2$ has been already determined.

3. Find the point $\bar{v} \in V^{s}$ that is most close to the point $v_{i}$, i.e. $\bar{v}=\arg \min _{v \in V^{s}}\left\|v-v_{i}\right\|$. Let the solution at $\bar{v}$ be $U^{*}(\bar{v})$.

4. Solve $\mathrm{P} 2$ at the point $v_{i}$ with initial guess for the optimization variables set to $U^{*}(\bar{v})$.

5. If a solution of $\mathrm{P} 2$ at the point $v_{i}$ has been found, mark $v_{i}$ as feasible and add it to the set $V^{s}$. Otherwise, mark $v_{i}$ as infeasible.

6. Let $i=i+1$. If $i \leq N_{1}$, go to step 2. Otherwise, terminate.

\section{B. Computation of feasible PWL solution}

Definition 1 (Feasibility on a discrete set):

Let $X=\left\{v_{1}, v_{2}, \ldots, v_{Q}\right\} \subset \mathbb{R}^{\tilde{n}}$ be a discrete set. A function
$U(\tilde{x})$ is feasible on $X$ if $G\left(U\left(v_{i}\right), v_{i}\right) \leq 0, i \in\{1,2, \ldots, Q\}$.

We restrict our attention to a hyper-rectangle $X \subset \mathbb{R}^{\tilde{n}}$ where we seek to approximate the optimal solution $U^{*}(\tilde{x})$ to problem $\mathrm{P} 2$. We require that the state space partition is orthogonal and can be represented as a $k-d$ tree. The main idea of the approximate mp-NLP approach is to construct a feasible piecewise linear (PWL) approximation $\hat{U}(\tilde{x})$ to $U^{*}(\tilde{x})$ on $X$, where the constituent affine functions are defined on hyper-rectangles covering $X$. In case of convexity, it suffices to compute the solution of problem P2 at the $2^{\widetilde{n}}$ vertices of a considered hyper-rectangle $X_{0}$ by solving up to $2^{\widetilde{n}}$ NLPs. In case of non-convexity, it would not be sufficient to impose the constraints only at the vertices of the hyper-rectangle $X_{0}$. One approach to resolve this problem is to include some interior points in addition to the set of vertices of $X_{0}$ [23]. These additional points can represent the vertices and the facets centers of one or more hyper-rectangles contained in the interior of $X_{0}$. Based on the solutions at all points, a feasible local linear approximation $\hat{U}_{0}(\tilde{x})=K_{0} \tilde{x}+g_{0}$ to the optimal solution $U^{*}(\tilde{x})$, valid in the whole hyper-rectangle $X_{0}$, is determined by applying the following procedure:

Procedure 3 (computation of explicit approximate solution):

Suppose A1 and A2 hold. Consider any hyper-rectangle $X_{0} \subseteq X_{f}$ with a set of points $V_{0}=\left\{v_{0}, v_{1}, v_{2}, \ldots, v_{N_{1}}\right\}$ determined by applying Procedure 1. Compute $K_{0}$ and $g_{0}$ by solving the following NLP:

Problem P3:

$$
\begin{aligned}
\min _{K_{0}, g_{0}} \sum_{i=0}^{N_{1}}\left(J\left(K_{0} v_{i}+g_{0}, v_{i}\right)-V^{*}\left(v_{i}\right)\right. \\
\left.+\beta\left\|K_{0} v_{i}+g_{0}-U^{*}\left(v_{i}\right)\right\|_{2}^{2}\right)
\end{aligned}
$$

subject to:

$$
G\left(K_{0} v_{i}+g_{0}, v_{i}\right) \leq 0, \forall v_{i} \in V_{0}
$$

In (30), the parameter $\beta>0$ is a weighting coefficient.

\section{Estimation of error bounds}

Suppose that a state feedback $\hat{U}_{0}(\tilde{x})$ that is feasible on $V_{0} \subseteq X_{0}$ has been determined by applying Procedure 3 . Then, for the cost function approximation error in $X_{0}$ we have:

$$
\varepsilon(\tilde{x})=\hat{V}(\tilde{x})-V^{*}(\tilde{x}) \leq \varepsilon_{0}, \tilde{x} \in X_{0}
$$

where $\hat{V}(\tilde{x})=J\left(\hat{U}_{0}(\tilde{x}), \tilde{x}\right)$ is the sub-optimal cost and $V^{*}(\tilde{x})$ denotes the cost corresponding to the close-to-global solution $U^{*}(\tilde{x})$, i.e. $V^{*}(\tilde{x})=J\left(U^{*}(\tilde{x}), \tilde{x}\right)$. The following 
procedure can be used to obtain an estimate $\hat{\varepsilon}_{0}$ of the maximal approximation error $\varepsilon_{0}$ in $X_{0}$.

Procedure 4 (computation of the error bound): Consider any hyper-rectangle $X_{0} \subseteq X_{f}$ with a set of points $V_{0}=\left\{v_{0}, v_{1}, v_{2}, \ldots, v_{N_{1}}\right\}$ determined by applying Procedure 1. Compute an estimate $\hat{\varepsilon}_{0}$ of the error bound $\varepsilon_{0}$ through the following maximization:

$$
\hat{\varepsilon}_{0}=\max _{i \in\left\{0,1,2, \ldots, N_{1}\right\}}\left(\hat{V}\left(v_{i}\right)-V^{*}\left(v_{i}\right)\right)
$$

\section{Approximate $m p-N L P$ algorithm for explicit GP- NMPC}

Assume the tolerance $\bar{\varepsilon}>0$ of the cost function approximation error is given. The following algorithm is proposed to design explicit reference tracking GP-NMPC:

Algorithm 1 (explicit reference tracking GP-NMPC)

1. Initialize the partition to the whole hyper-rectangle, i.e. $\Pi=\{X\}$. Mark the hyper-rectangle $X$ as unexplored.

2. Select any unexplored hyper-rectangle $X_{0} \in \Pi$. If no such hyper-rectangle exists, terminate.

3. Compute a solution to problem $\mathrm{P} 2$ at the center point $v_{0}$ of $X_{0}$ by applying Procedure 2a. If $\mathrm{P} 2$ has a feasible solution, go to step 4 . Otherwise, split $X_{0}$ into two hyperrectangles $X_{1}$ and $X_{2}$ by applying the heuristic rule 2 from [23]. Mark $X_{1}$ and $X_{2}$ unexplored, remove $X_{0}$ from $\Pi$, add $X_{1}$ and $X_{2}$ to $\Pi$, and go to step 2 .

4. Define a set of points $V_{0}=\left\{v_{0}, v_{1}, v_{2}, \ldots, v_{N_{1}}\right\}$ by applying Procedure 1. Compute a solution to problem P2 for $\tilde{x}$ fixed to each of the points $v_{i}, i=1,2, \ldots, N_{1}$ by applying Procedure $2 \mathrm{~b}$. If $\mathrm{P} 2$ have a feasible solution at all these points, go to step 6 . Otherwise, go to step 5 .

5. Compute the size of $X_{0}$ using some metric. If it is smaller than some given tolerance, mark $X_{0}$ infeasible and explored and go to step 2. Otherwise, split $X_{0}$ into hyperrectangles $X_{1}, X_{2}, \ldots, X_{N_{s}}$ by applying the heuristic rule 1 from [23]. Mark $X_{1}, X_{2}, \ldots, X_{N_{s}}$ unexplored, remove $X_{0}$ from $\Pi$, add $X_{1}, X_{2}, \ldots, X_{N_{s}}$ to $\Pi$, and go to step 2 .

6. Compute an affine state feedback $\hat{U}_{0}(\tilde{x})$ using Procedure 3, as an approximation to be used in $X_{0}$. If no feasible solution was found, split $X_{0}$ into two hyperrectangles $X_{1}$ and $X_{2}$ by applying the heuristic rule 3 from [23]. Mark $X_{1}$ and $X_{2}$ unexplored, remove $X_{0}$ from $\Pi$, add $X_{1}$ and $X_{2}$ to $\Pi$, and go to step 2 .

7. Compute an estimate $\hat{\varepsilon}_{0}$ of the error bound $\varepsilon_{0}$ in $X_{0}$ by applying Procedure 4 . If $\hat{\varepsilon}_{0} \leq \bar{\varepsilon}$, mark $X_{0}$ as explored and feasible and go to step 2. Otherwise, split $X_{0}$ into two hyper-rectangles $X_{1}$ and $X_{2}$ by applying Procedure 4 from [23]. Mark $X_{1}$ and $X_{2}$ unexplored, remove $X_{0}$ from $\Pi$, add $X_{1}$ and $X_{2}$ to $\Pi$, and go to step 2 .

\section{Simulation EXAMPle}

\section{A. The nonlinear system}

Consider the stochastic system described by the following nonlinear state space model:

$$
\dot{x}=-\tanh \left(x+u^{3}\right)+\xi_{1}
$$

where $\xi_{1}$ is white noise with variance 0.0025 and zero mean. The sampling time, determined according to system dynamics, was selected to be $T_{s}=0.5$. The Euler approximation of system (34) is:

$$
x(t+1)=x(t)-T_{s} \tanh \left(x(t)+u(t)^{3}\right)+\xi_{2}(t)
$$

where $\xi_{2}(t)=T_{s} \xi_{1}(t)$.

\section{B. Gaussian process model identification}

The control signal $u$ was generated by a random number generator with normal distribution. The control signal blocking was $T_{u}=6 T_{s}$, i.e. it is kept constant for 6 time instants. The number $M$ of the input signal samples used for the identification determines the dimension of the covariance matrix. In our case, $M=200$. Let $x_{m}$ be the mean value of the state of system (35) obtained for the generated control signals, i.e. $x_{m}=\frac{1}{M} \sum_{1}^{M} x(t)$. By introducing the variable $y(t)=x(t)-x_{m}$, we would like to obtain a Gaussian process model for the following discretetime stochastic system:

$$
y(t+1)=y(t)-T_{s} \tanh \left(y(t)+u(t)^{3}\right)+\xi_{2}(t)
$$

Based on the generated data set, the discrete-time system (36) is approximated with Gaussian process with zero mean and covariance function of the form (1). The maximum likelihood framework was used to determine the hyperparameters. The optimization method applied for identification of the Gaussian process model was the conjugate gradient method with line searches [28]. The following set of hyperparameters was found:

$$
\Theta=\left[w_{1}, w_{2}, v_{0}, v_{1}\right]=[0.3952,0.9754,1.0333,0.0354] \text { (37) }
$$

A validation control input signal was generated by random number generator with normal distribution and rate of change that is different from the one used for the identification signal. The response of the Gaussian process model to the validation signal is shown in Fig. 1. The associated average squared error and log density error are respectively $A S E=0.0017$ and $L D=-2.1476$. 




Fig. 1. Response of the Gaussian process model to the excitation signal used for validation.

\section{Design of explicit reference tracking GP-NMPC controller}

The mp-NLP approach described in section IV is applied to design an explicit reference tracking GP-NMPC controller for the system (35) based on the obtained Gaussian process model.

In the GP-NMPC problem formulation (problem P1), the predicted state $x_{t+k+1 \mid t}$ of system (35) is used. This prediction is obtained in the following way. First, we obtain the prediction of $y_{t+k+1 \mid t}$ from the Gaussian process model of system (36):

$$
\begin{aligned}
& y_{t+k+1 \mid t} \mid y_{t+k \mid t}, u_{t+k} \sim \mathcal{N}\left(\mu\left(y_{t+k+1 \mid t}\right), \sigma^{2}\left(y_{t+k+1 \mid t}\right)\right) \\
& k=0,1, \ldots, N-1
\end{aligned}
$$

Then, the predicted $x_{t+k+1 \mid t}$ is:

$$
x_{t+k+1 \mid t}=y_{t+k+1 \mid t}+x_{m}
$$

The iterative multi-step ahead prediction was done by feeding back at each time step the predictive mean only.

The following control input and rate constraints are imposed on the system:

$$
-1 \leq u \leq 1 ;-0.5 \leq \Delta u \leq 0.5
$$

The prediction horizon is $N=8$ and the terminal constraint is:

$$
\begin{aligned}
& \max \left\{\left\|\mu\left(x_{t+N \mid t}\right)-2 \sigma\left(x_{t+N \mid t}\right)-r(t)\right\|,\right. \\
& \left.\left\|\mu\left(x_{t+N \mid t}\right)+2 \sigma\left(x_{t+N \mid t}\right)-r(t)\right\|\right\} \leq \delta
\end{aligned}
$$

where $\delta=0.015$. The weighting matrices in the cost function (21) are $Q=10, R=1, P=10$. The GP-NMPC minimizes the cost function (21) subject to the Gaussian process model (38)-(39) and the constraints (40), (41).

The formulated GP-NMPC problem results in optimization problem $\mathrm{P} 2$ with 8 optimization variables and 33 constraints. One internal region $X_{0}^{1} \subset X_{0}$ is used in Procedures 1, 2, 3 and 4. This results in problem P3 which has 32 optimization variables and 285 constraints. In (30), it is chosen $\beta=10$. The approximation tolerance is chosen in the following way:

$$
\bar{\varepsilon}\left(X_{0}\right)=\max \left(\bar{\varepsilon}_{a}, \bar{\varepsilon}_{r} \min _{\tilde{x} \in X_{0}} V^{*}(\tilde{x})\right),
$$

where $\bar{\varepsilon}_{a}=0.005$ and $\bar{\varepsilon}_{r}=0.05$ are the absolute and the relative tolerances, respectively. The extended state vector is $\tilde{x}(t)=[x(t), r(t), u(t-1)] \in \mathbb{R}^{3}$, which leads to a 3dimensional state space to be partitioned. The latter is defined by $X=[-1.2,1.2] \times[-0.7,0.7] \times[-1,1]$.

The partition has 1419 regions and 18 levels of search. Totally, 24 arithmetic operations are needed in real-time to compute the control input (18 comparisons, 3 multiplications and 3 additions).

The performance of the closed-loop system was simulated for the following set point change:

$$
\begin{aligned}
& r(t)=-0.5, t \in[0 ; 50] ; r(t)=-0.2, t \in[51 ; 100] \\
& r(t)=0.2, t \in[101 ; 150] ; r(t)=0.5, t \in[151 ; 200]
\end{aligned}
$$

and initial conditions for the state and control variable $x(0)=0$ and $u(0)=0$, respectively. The resulting closedloop response is depicted in Fig. 2 to Fig. 4.

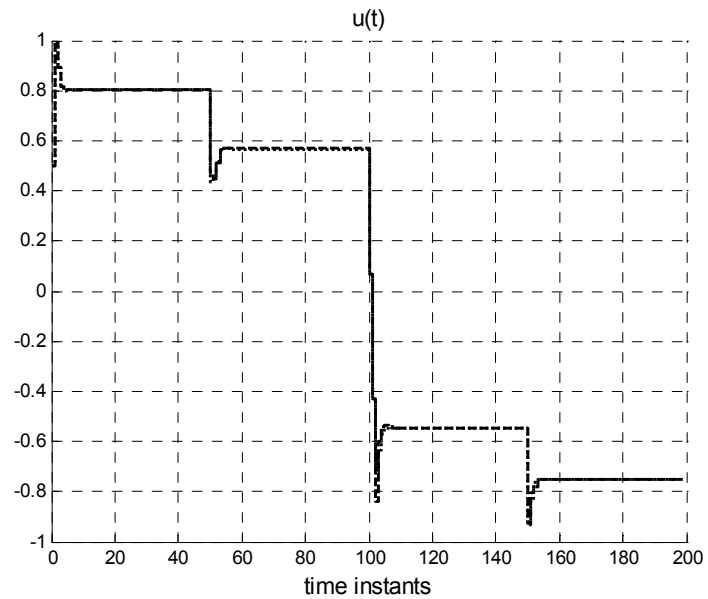

Fig. 2. The control input. The dashed curve is with the approximate explicit GP-NMPC and the dotted curve is with the exact GP-NMPC.

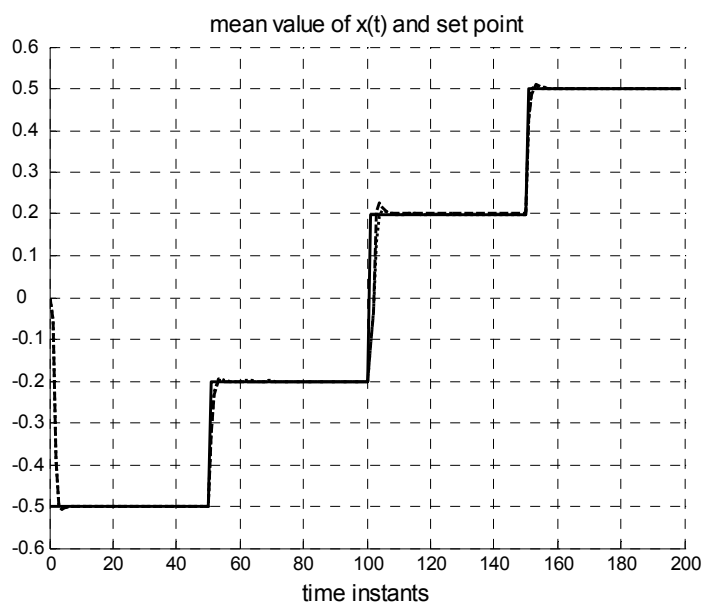

Fig. 3. The mean value of the state variable predicted with the Gaussian process model. The dashed curve is with the approximate explicit GPNMPC, the dotted curve is with the exact GP-NMPC, and the solid curve is the set point. 


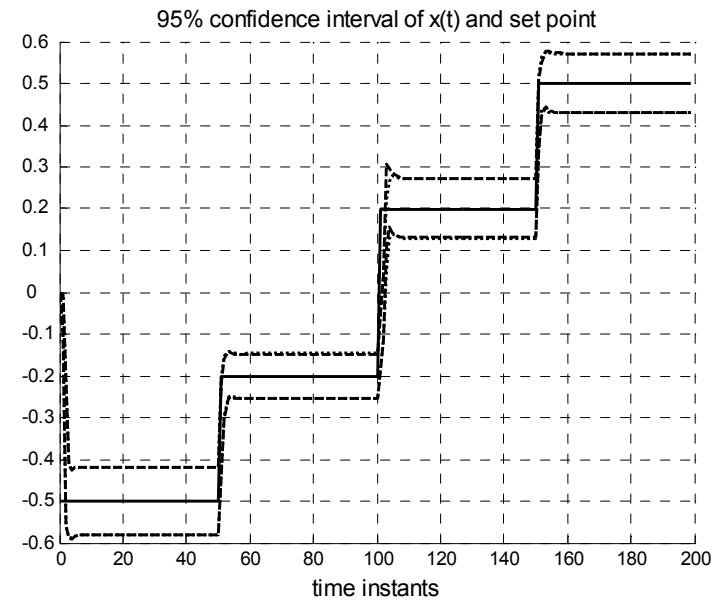

Fig. 4 . The $95 \%$ confidence interval of the state variable predicted with the Gaussian process model. The dashed curve is with the approximate explicit GP-NMPC, the dotted curve is with the exact GP-NMPC, and the solid curve is the set point.

The results show that the exact and the approximate solutions are almost indistinguishable.

\section{CONCLUSIONS}

In this paper, an approximate mp-NLP approach to explicit solution of reference tracking NMPC problems based on Gaussian process models is developed. The approach builds an orthogonal search tree structure of the state space partition and consists in constructing a feasible PWL approximation to the optimal control sequence.

\section{REFERENCES}

[1] S. S. Keerthi and E. G. Gilbert, "Optimal infinite horizon feedback laws for a general class of constrained discrete-time systems: Stability and moving horizon approximations", Journal of Optimization Theory and Applications, vol. 57, pp. 265-293, 1988.

[2] D. Q. Mayne, J. B. Rawlings, C. V. Rao, and P. O. M. Scokaert, "Constrained model predictive control: Stability and optimality", Automatica, vol. 36, pp. 789-814, 2000.

[3] F. Allgöwer and A. Zheng (eds.), Nonlinear Model Predictive Control, Progress in system and control theory, Vol. 26, Birkhäuser Verlag, Basel, 2000.

[4] B. Kouvaritakis and M. Cannon (eds.), Nonlinear predictive control, Theory and practice, IEE Control Engineering Series 61, IEE, 2001.

[5] L. Magni and R. Scattolini, "Stabilizing model predictive control of nonlinear continuous time systems", Annual Reviews in Control, vol. 28, pp. 1-11, 2004.

[6] J. H. Lee and B. L. Cooley, "Optimal feedback control strategies for state-space systems with stochastic parameters", IEEE Transactions on Automatic Control, vol. 43, No. 10, pp. 1469-1475, 1998.

[7] D. van Hessem, C. W. Scherer, and O. H. Bosgra, "LMI-based closed-loop economic optimization of stochastic process operation under state and input constraints. Proceedings of the 40-th IEEE Conference on Decision and Control, 2001, pp. 4228-4233.

[8] J. Yan and R. R. Bitmead, "Incorporating state estimation into model predictive control and its application to network traffic control", Automatica, vol. 41, pp. 595-604, 2005.

[9] B. Kouvaritakis, M. Cannon, and P. Couchman, "MPC as a tool for sustainable development integrated policy assessment", IEEE Transactions on Automatic Control, vol. 51, No. 1, pp. 145-149, 2006.

[10] P. Couchman, B. Kouvaritakis, and M. Cannon, "LTV models in MPC for sustainable development", International Journal of Control, vol. 79, No. 1, pp. 63-73, 2006.
[11] P. Couchman, M. Cannon, and B. Kouvaritakis, "Stochastic MPC with inequality stability constraints", Automatica, vol. 42, No. 12, pp. 2169-2174, 2006.

[12] P. Couchman, M. Cannon, and B. Kouvaritakis, "MPC for stochastic systems", Proceedings of the International Workshop on Assessment and Future Directions of Nonlinear Model Predictive Control, Freudenstadt-Lauterbad, Germany, 26-30 August, 2005, pp.61-72.

[13] J. Kocijan, A. Girard, B. Banko, and R. Murray-Smith, "Dynamic systems identification with Gaussian processes", Mathematical and Computer Modelling of Dynamic Systems, vol. 11, No. 4, pp. 411424, 2005.

[14] E. Solak, R. Murray-Smith, W. E. Leithead, D. J. Leith, and C. E. Rasmussen, "Derivative observations in Gaussian process models of dynamic systems", NIPS 15, Vancouver, Canada, MIT Press, 2003.

[15] A. Girard, C. E. Rasmussen, J. Quinonero Candela, and R. MurraySmith, "Gaussian process priors with uncertain inputs \& application to multiple-step ahead time series forecasting", NIPS 15, Vancouver, Canada, MIT Press, 2003.

[16] J. Kocijan, and R. Murray-Smith, "Non-linear predictive control with a Gaussian process model", In Switching and Learning in Feedback Systems, Eds. R. Shorten and R. Murray-Smith, Lecture Notes in Computer Science 3355, Springer-Verlag, Heidelberg, Germany, 2005.

[17] B. Likar and J. Kocijan, "Predictive control of a gas-liquid separation plant based on a Gaussian process model", Computers \& Chemical Engineering, vol. 31, pp. 142-152, 2007.

[18] R. Murray-Smith, D. Sbarbaro, C. E. Rasmussen, and A. Girard, "Adaptive, cautious, predictive control with Gaussian process priors", Proceedings of 13-th IFAC Symposium on System Identification, Rotterdam, 2003.

[19] A. Bemporad, M. Morari, V. Dua, and E. N. Pistikopoulos, "The explicit linear quadratic regulator for constrained systems". Automatica, vol. 38, pp. 3-20, 2002.

[20] M. Bacic, M. Cannon, and B. Kouvaritakis, "Constrained NMPC via state-space partitioning for input-affine non-linear systems". Proceedings of American Control Conference, Denver, Colorado, 2003.

[21] T. A. Johansen, "On multi-parametric nonlinear programming and explicit nonlinear model predictive control", Proceedings of IEEE Conference on Decision and Control, Las Vegas, NV, 2002, vol. 3, pp. 2768-2773.

[22] T. A. Johansen, "Approximate explicit receding horizon control of constrained nonlinear systems", Automatica vol. 40, pp. 293-300, 2004.

[23] A. Grancharova, T. A. Johansen, and P. Tøndel, "Computational aspects of approximate explicit nonlinear model predictive control", Proceedings of the International Workshop on Assessment and Future Directions of Nonlinear Model Predictive Control, FreudenstadtLauterbad, Germany, 26-30 August, 2005, pp. 85-96.

[24] A. V. Fiacco, Introduction to sensitivity and stability analysis in nonlinear programming. Orlando, Fl: Academic Press, 1983.

[25] T. A. Johansen and A. Grancharova, "Approximate explicit constrained linear model predictive control via orthogonal search tree", IEEE Transactions on Automatic Control, vol. 48, pp. 810-815, 2003.

[26] C. K. I. Williams, "Prediction with Gaussian processes: From linear regression to linear prediction and beyond", In Learning in Graphical Models, M.I. Jordan, (ed.), Kluwer Academic, Dordrecht, pp. 599621, 1998.

[27] C. E. Rasmussen, Evaluation of Gaussian Processes and other Methods for Non-Linear Regression, Ph.D. Disertation, Graduate department of Computer Science, University of Toronto, Toronto, 1996.

[28] A. Girard and R. Murray-Smith, "Gaussian process: Prediction at a noisy input and application to iterative multiple-step ahead forecasting of time-series", In Switching and Learning in Feedback Systems, Eds. R. Shorten and R. Murray-Smith, Lecture Notes in Computer Science 3355, Springer-Verlag, Heidelberg, Germany, 2005. 